\title{
Designing a Hardware in the Loop Wireless Digital Channel Emulator for Software Defined Radio
}

\author{
Janarbek Matai*, Pingfan Meng*, Lingjuan Wu*, Brad Weals ${ }^{\dagger}$, and Ryan Kastner* \\ ${ }^{*}$ Department of Computer Science and Engineering, University of California, San Diego \\ ${ }^{\dagger}$ Toyon Research Corporation \\ \{jmatai, pmeng, 11wu, kastner\}@cs.ucsd.com, bweals@toyon.com
}

\begin{abstract}
The testing, verification and evaluation of wireless systems is an important but challenging endeavor. The most realistic method to test a wireless system is a field deployment. Unfortunately, this is not only expensive but also time consuming. In this paper, we present the design and implementation of a digital wireless channel emulator, which connects directly to a number of radios, and mimics the wireless channels between them, across a range of scenarios, in real-time. We use high-level synthesis tools to design the emulator while performing design space exploration. We describe the optimizations and tradeoffs that were necessary to reach the target throughput and area requirements.
\end{abstract}

\section{INTRODUCTION}

One of the many challenges in the development and evaluation of wireless systems is testing and verification. Ultimately, radio systems need to be field tested to ensure that they satisfy the stated requirements. However, this is expensive, time consuming, and difficult to repeat. Simulation can overcome these problems, but is ultimately limited by fidelity issues and/or excessive run times; thus it is more suited to the early stages of development. Real-time hardware in the loop (HWIL) radio frequency (RF) channel emulation fills the gap left between simulation and field testing. A good RF channel emulator will provide repeatability, high-fidelity, and the opportunity to test complete radio systems in different network scenarios. This is especially important for state-of-the-art wireless systems which incorporate complex link adaptation algorithms with the goals of improving spectral efficiency, reducing inter-symbol interference (ISI), and increasing resilience against multipath, Doppler shift, and fading.

Software defined radios (SDR) use agile spread spectrum, ultra wideband and other techniques to cognitively utilize the available spectrum. They can operate over different frequency band and have been proposed for next generation military and civilian voice, video and data links. In order to evaluate the effectiveness of SDR networks in responding to adverse link conditions, an appropriate real-time, accurate RF channel emulator is required. A channel emulator simulates the antenna and propagation of the signal, modifying it depending upon a predetermined scenario. It works directly with a variety of existing radios, and thus must handle a wide range of frequencies with rapidly varying channels over a range of distances.
To meet the challenging requirements in testing and evaluating the state-of-the-art wireless systems, we look at a portion of this overall system. We designed a digital wireless channel emulator (DWCE) on an FPGA platform. This is high fidelity and broadband. It provides real-time emulation for radios operating in a frequency range of $2 \mathrm{MHz}$ to $2 \mathrm{GHz}$. It has a range resolution of $0.25 \mathrm{~km}$. It also provides link losses of up to $130 \mathrm{~dB}$ and has a resolution of $0.5 \mathrm{~dB}$.

A major focus of this work is to evaluate the suitability of implementing our DWCE using the AutoESL high-level synthesis (HLS) tool. Our DWCE has to handle wide range of dynamically changing parameters such as Doppler effect fast fading, and multipath. We carefully describe the optimization process that we used to obtain a design that meets the required specification requirements. This was done solely through modification to the code given to the HLS tool. And only through careful optimization of this code, with an understanding of how this code effect the HLS process, were we able to meet the required performance metrics. The DWCE code is significant, consisting of over 700 lines of $\mathrm{C}$ code. Thus it provides an interesting example of a complex, high throughput design using high-level synthesis tools targeting FPGAs. We show how to effectively use the HLS tools to achieve the target design goal.

The specific contributions of this paper include:

1) Designing an FPGA implementation of a wireless channel emulator operating across a $2 \mathrm{MHz}-2 \mathrm{GHz}$ spectrum with a range resolution of $0.25 \mathrm{~km}$ and link losses up to $130 \mathrm{~dB}$ with $0.3 \mathrm{~dB}$ resolution.

2) Studying the effectiveness of the AutoESL HLS tool to design a complex, high throughput application. The emulator has three major modules, PathDelays, PathGains, and ChannelFunction with over 180, 500, 120 lines of code, respectively. Each module has $C \times M^{2}$ complexity where $M$ is the number of paths in each channel and $C$ is the number of loops in each module.

3) Describing the benefits of different optimization techniques on the area and throughput of the emulator. Starting with a naive implementation, we utilize and analyze a number of optimizations in order to reach to target performance metrics.

The remainder of this paper is organized as follows: We 
provide an overview of related work in Section II. Section III describes the wireless channel emulator model in detail. In Section IV, we give detailed description of hardware design and implementation of wireless channel emulator. Section V presents experimental results and we conclude in Section VI.

\section{RELATED WORK}

There are several previously published wireless channel simulators and emulators using an FPGA [1], [2], [3]. Borries et al. [1] focused on stationary 802.11 laptop type wireless connections. Our emulator is looking at broader scope of networks by modeling longer and dynamically varying channel delays, Doppler effects and fast fading. This requires accounting for multipath, Doppler effects, and link loss. The emulator by Murphy et al. [2] uses a Nakagami fading model and accounts for Doppler effects. However, they do not handle continuously and smoothly varying delays. And while their emulator can model a Doppler shift, it is an approximation of the Doppler effect. In case where we do not have to up and down convert, Doppler scaling is handled completely by the continuous delay model. If we do have to down and upconvert, then that delay model in combination with our Doppler shift correction gives us the complete Doppler Effect.

Buscemi et al. presented study of scalability of digital wireless channel emulators using cluster of FPGAs [3]. They implemented tapped delay line (TDL) channel model in VHDL and prototyped it on a two node FPGA system. In our opinion, the major contribution of the work is the system design of many DWCE across an FPGA based compute cluster. Our work differs in two ways. First, we implement statistical wireless channel model; the channel model that we use is significantly more complicated with respect to implementation. It is outside the scope of this paper to argue whether the channel model that we use is better, but it is clear that it is more complex (in terms of hardware implementation) and thus makes for a more interesting HLS case study. Secondly, we focus more on the design of the DWCE using HLS tools rather than the overall system design. Our DWCE could be used within the system that they propose.

Our FPGA implementation is similar to the wireless channel simulator developed by Iskander et al. [4]. They implemented multipath fading channel simulator in Matlab. Our emulator is several thousand times faster than the simulator.

There is a substantial amount of research around the use of high-level synthesis tools to implement a wide variety of applications on FPGAs [5]. AutoESL is used to implement applications including compressive sensing [6], stereo matching [7], a sphere detector for broadband wireless systems [8], and real-time HDTV [9]. Catapult-C has been used to implement 64-QAM decoder [10], and a sphere decoder [11]. ROCCC is used to design a Smith-Waterman algorithm [12] and a molecular dynamics algorithm [13]. Impulse $\mathrm{C}$ is used to implement a tomographic reconstruction algorithm [14].

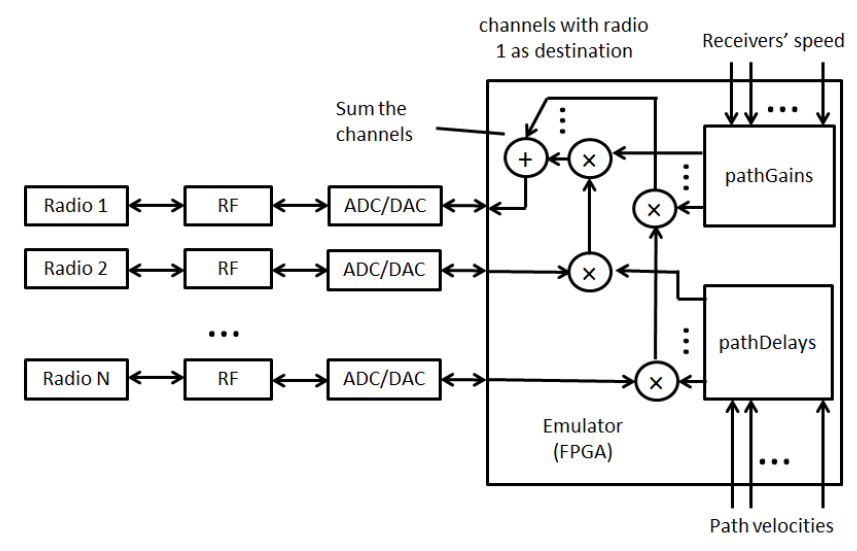

Fig. 1. System overview of the wireless channel emulator.

\section{WiRELESS ChANNEL Model}

This section provides a description of the wireless channel model and algorithms that our emulator uses.

Figure 1 provides a graphical description of the proposed channel emulator. The emulator connects to $N$ radios using analog-to-digital (ADC) and digital-to-analog converters (DAC) in series with an RF module. The radios connect to the RF module in lieu of an antenna. The RF module down converts the radio signal to baseband signal. Then the ADC converts the baseband signal to digital signal as the input to the emulator. The emulator combines all the digital signal inputs and processes them with the wireless channel model (described in the next subsection) to produce the digital outputs for each radio. The digital output signals are converted to an analog signal by the DAC and up converted to radio frequency by the RF module. Finally, each radio receives an RF signal as if it is in a real field testing environment with $N-1$ other radios. We designed the emulator to accept signals within a $2 \mathrm{MHz}$ to $30 \mathrm{MHz}$ range.

The wireless channel can be broken down into a set of receive/transmit paths. For example, an emulator with 8 radio ports has up to 56 channels with the assumption that the receive/transmit paths are non-symmetric. A transmitted RF signal encounters reflection, diffuse scattering, and diffraction due to the ground and the obstacles in the environment. There is also a Doppler effect which is caused by the motion of the transmitter and the receiver. The emulator is responsible for calculating these environment effects in the digital signal domain.

A single wireless channel is described in Eq (1), where $s i_{t-i \Delta \tau}$ are the previous $n$ input complex samples, $w(t)_{i}$ is a dynamically changing set of complex weights, and $s o_{t}$ is the complex output of the channel at the present sample time. The weights $w(t)_{i}$ control the delays and the gains of output signals to emulate the environment effects of the simulated scenario. 


$$
s o_{t}=\sum_{i=0}^{n} s i_{t-i \Delta \tau} w(t)_{i}
$$

The methods PathDelays and PathGains are used in tandem to compute $w(t)_{i}$. PathDelays emulates the real field testing path delay phenomenon by integrating the path velocity. In this model, we use tapped delay line (TDL) model. A TDL model stores input samples in a FIFO. Then, it computes the delay of ADC samples in the FIFO. The output of PathDelays is a series of sample numbers from the delay line representing the first ADC sample of a consecutive group for each path and a series of weight vectors for each path. In order to access the samples in the delay line, PathDelays calculates the index for each path. Thus, the output of PathDelays is index[N] and weight [K][N] where $N$ is the number of paths and $K$ is predefined constant.

PathGains emulates the simulated scenario's path gain. It implements a statistical method to model the effect of many independent scatters near the receiver where none of them is dominant. PathGains filters a complex white noise source for each path. The result of the filter represents the path gain considering the effect of reflections, obstacles, etc. PathGains also computes the Doppler effect by controlling the coefficients of the white noise filter. The result of PathGains is a series of complex gains used to multiply the output digital signals of PathDelays. The output signal of PathGains is equivalent to the signal as it is transmitted across a real fading environment. In our model, we use complex signals. Thus the output of PathDelays is the matrix gains[2][N]. After getting, index[N], weight $[K][N]$, and gains[2][N], we can calculate real and imaginary part of complex sample output using Eqs (4) and (5)

$$
\begin{gathered}
\text { tapline }_{i j}=\text { delayline }_{\text {index }} \\
\text { taps }_{i}=\sum_{i, j=0}^{N, K} \text { tapline }_{i j} * \text { weight }_{j i} \\
\text { so_r } r_{i}=\sum_{i=0}^{N} \text { gains_r }_{i} * \text { taps_r } r_{i}-\text { gains_ } i_{i} * \text { taps_ } i_{i} \\
\text { so_ } i_{i}=\sum_{i=0}^{N} \text { gains_r }_{i} * \text { taps_ } i_{i}-\text { gains_ } i_{i} * \text { taps_r } r_{i}
\end{gathered}
$$

where $g a i n s \_r, t a p s \_r$ and gains_ $i, t a p s \_i$ are the real and imaginary part of the complex signals for each path.

As shown above, each channel between the various radios are modeled as described in Equation (1) which is further describe in Equations (2), (3), (4), (5). The remainder of the paper focuses on implementing a single channel emulator. This can be duplicated to model $N$ channels.

\section{HARdWARE DESIGN AND Optimization}

In this section, we introduce the architecture of the emulator and discuss the design process using AutoESL. AutoESL is a high-level synthesis tool from Xilinx. It takes as input synthesizable $\mathrm{C} / \mathrm{C}++/$ SystemC code. Due to space limitation,

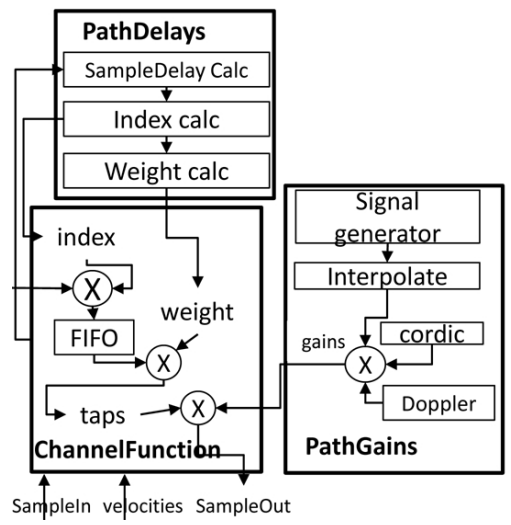

Fig. 2. A block diagram of the channel emulator, which includes three main functions ChannelFunction, PathGains and PathDelays.

we skip to cover AutoESL optimization pragmas and refer [7] for more detail. More specifically, we provide an algorithmic description of the emulator, and present how different optimization of AutoESL affects area (BRAM, DSP48E, FF, LUT) and latency. We present four different results of different design optimizations (Baseline, Restructured, Bit-width, and PUP).

\section{A. Architecture}

The block diagram of the system is shown in Figure 2. It consists of three modules: PathDelays, PathGains and ChannelFunction.

ChannelFunction accepts an input signal, path velocities, and carrier frequency, and calculates the complex output sample signal. It sends path velocities to PathDelays and gets the index of starting signal and weight. The index is used to retrieve the delayed signal samples from the input signal as described in Eq (2). The results are used to calculate taps for each path using Eq (3). The samples from the input signal, weight and gain from PathGains are used to calculate the complex signal (SampleOut) as described in Eqs (4) and (5).

The PathDelays module takes as input the path velocities of each path in a channel. It computes the indexes of the input samples and the weights to calculate the expected sample at the specified path distance for all paths. It has three sub blocks as shown in Figure 2. Each sub block depends on results from the previous. It outputs vectors index[N] and weight [K][N]. PathDelays and ChannelFunction require a throughput of 2-30 $\mathrm{MHz}$ in order for the emulator to meet the required operating characteristics.

PathGains generates a random signal, interpolates the signal with a filter, and calculates the gain by multiplying the Doppler effect, interpolation and CORDIC outputs. PathGains has a throughput requirement of $100 \mathrm{kHz}-200 \mathrm{kHz}$ to meet the operating characteristics. The result matrix gain[2][N] is integrated with every 80 results of PathDelays.

We started with Matlab source code for the three modules. We converted these to synthesizable $\mathrm{C}$ code, e.g., limited pointers, no dynamic memory, and writing our own standard $\mathrm{C}$ 


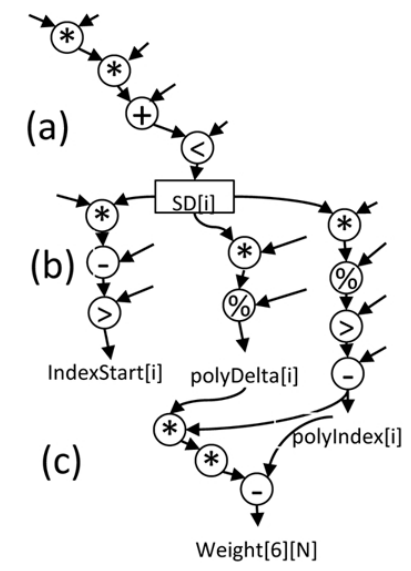

Fig. 3. The final restructured PathDelays code. There are three blocks: (a) sample delay calculation, (b) index calculation, (c) weight calculation.

functions ( $\max , \min$, pow, sin, cos). Then, we synthesized the functions with AutoESL. We achieved the target design goal using the following steps: code restructuring, bit-width optimization, and pipelining, unrolling and memory partitioning. These steps are discussed in detail in the following.

\section{B. Baseline}

The baseline architecture uses the synthesizable $\mathrm{C}$ code "as is" and implemented it with AutoESL. Additionally, we created testbenches and top modules for each of PathDelays, PathGains and ChannelFunction. The primary goal of this step is to get an initial, functionally correct design with AutoESL. Typically, the area of design is large; it is not uncommon for this to be millions of times larger than the area of final optimized version. The throughput at this stage can be worse than original software implementation due to large clock period difference between CPU and FPGA. Additionally the manual implementation of standard $\mathrm{C}$ functions may incur additional cost since the standard $\mathrm{C}$ functions are highly optimized. To wit, the baseline design of PathDelays, PathGains and ChannelFunction are 106, 17, 4.8 times slower than their equivalent software implementations as in Figure 9. Again, the goal here is to make sure that the translated synthesizable $\mathrm{C}$ code is functionally correct. Once we have this, we perform optimizations that can significantly improve both the area and throughput.

In the following, we briefly describe the baseline implementations of PathDelays, PathGains and ChannelFunction.

PathDelays: This module calculates the sample delay by multiplying the path velocity by a constant for each of the $N$ paths. Then the maximum of sample delays and pre-computed index is selected. This procedure is shown in Figure 3 (a). Then using the previous sample delay, it calculates the indexStart, polyDelta and polyIndex as shown in Figure 3 (b). Finally, it calculates the weight for each path as shown in Figure 3 (c). The initial PathDelays function had seven blocks of code where each block is a loop. The result of each block

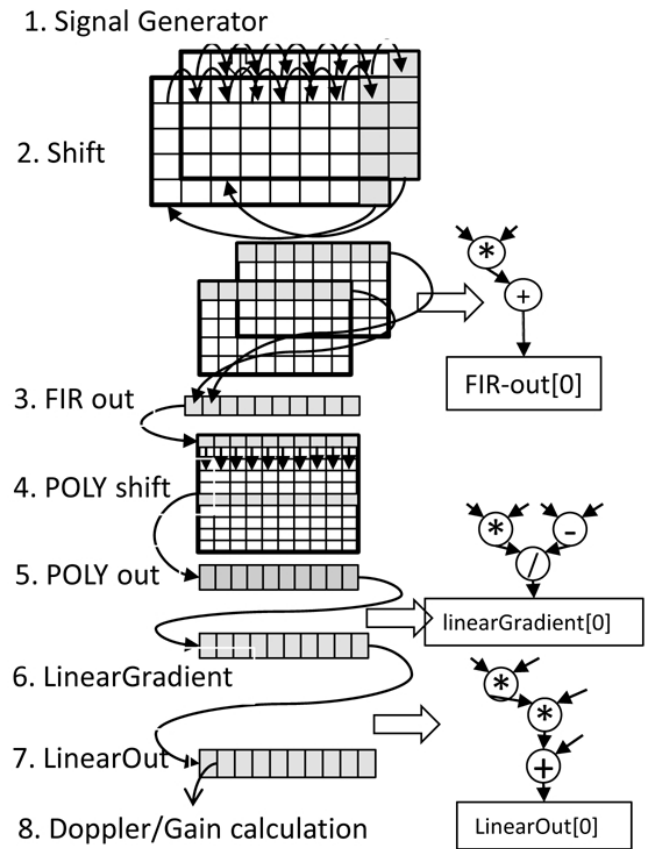

Fig. 4. The computation involved in the PathGains module.

is used in the subsequent block.

PathGains: This module has several steps. The first step involves signal generation through interpolation and Doppler effect calculation. Initially, it generates a random signal based on a linear feedback shift register (LFSR). Then the signal is filtered by FIR and POLY phase shaping filters to perform a linear interpolation. In the signal generation, we generate $N x M x K$ random values where $N$ is the number of paths, $M=256$ ( $M$ is fixed and same as number of elements in Jake's spectrum), and $K=2$ since we need to generate a value for a complex signal (real and imaginary parts). The final interpolation is a matrix of size $1 x N$. The interpolation results are used to calculate the gain for each path. Figure 4 shows the signal generator, interpolation and Doppler/gain calculation. In the Figure 4, Step 1 is signal generation, Steps 2-7 are interpolation and Step 8 is Doppler and gain calculation. To implement the base code for PathGains module, we converted 216 lines of Matlab code to 700 lines of $\mathrm{C}$. Then we removed loops that have variable number of bounds. In one case, we used the TRIP_COUNT directive to describe the minimum and maximum range of the loop. We wrote code for the standard $\mathrm{C}$ functions. For some functions, we removed them from design since they are use only few times with constant inputs, e.g., pow $(2,32)$ was replaced with constant.

ChannelFunction: This module did not require much optimization.

\section{Code Restructuring}

The goal of this stage is to perform optimization on the code itself without using AutoESL directives. In this step, we performed two optimizations; 1) loop merging, unrolling and loop flattening, and 2) removing computations that can 


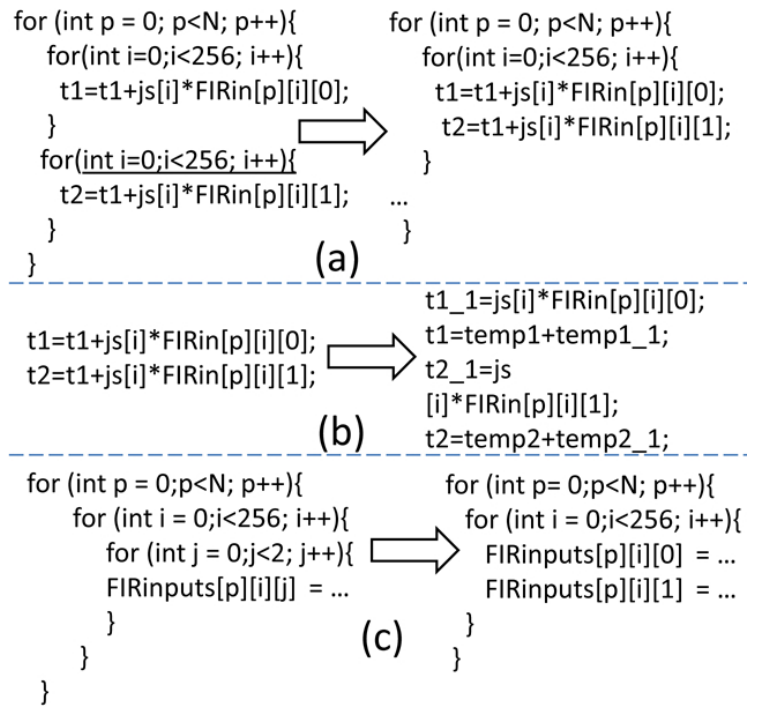

Fig. 5. Optimizations on the PathGains module including: (a) Loop merging, (b) Expression balancing, (c) Loop unrolling).

be done offline. Loop merging, unrolling and loop flattening saves clock cycles if used properly. AutoESL also provides directives to merge/flatten loops automatically.

As discussed in the previous section, we implemented some of standard $\mathrm{C}$ functions in baseline stage. In the design, some of those functions are used for a limited number of times. For example, we are using pow function 11 times in PathDelays module with constant input. Therefore, replaced these with a constant.

We describe the code restructuring optimizations for each of the modules in the following.

PathDelays: We optimized PathDelays in software using loop merging and removing functions that can be calculated offline. First, we removed two of the seven blocks because they are doing initialization. Then we did loop merging to reduce the number of blocks to three. Figure 3 shows the final structured code of PathDelays. Each block uses data from the previous block. Therefore, the optimal hardware will implement this design in three clock cycles. We unrolled the loops in each block as shown in Figure 3. Unrolled loops decreased number of clock cycles, however, this is limited by the access to the array. In AutoESL, arrays are implemented as block ram. Code restructuring of PathDelays reduced BRAMs, FF and LUT usage by 8 to 5(36\%), 7581 to 4070 (46\%) and 10170 to $4631(54 \%)$ respectively. The number of clock cycles decreased from 12430 to 2000 due to loop merging and removing some of the initialization step to offline. Decreased number of clock cycles resulted in increase in throughput drastically by $83 \%$. The code size reduced from 300 lines to 180 lines.

PathGains: The initial code had 17 blocks. Figure 4 shows most but not all of these blocks. Each block is embedded inside a loop. 4 of 17 blocks are responsible for $80 \%$ of computation. These are blocks 1, 2, 3 and 8 in Figure 4. Block 1 initializes an $N \times 256 \times 2$ array with random values from a linear feedback shift register. Blocks 2 and 3 perform shifting and FIR filtering operations. Block 8 applies the Doppler effect and calculates the gain for each path. We focused on these blocks and performed loop merging, expression balancing, and loop unrolling. In Figure 5, we presented some of the optimizations. In Figure 5 (a), we merged two loops. This is a snippet of code for FIR output calculation. This merging reduced the number of clock cycles from 50215 to 41250 , a $20 \%$ reduction. Then, we performed expression balancing and loop unrolling as shown in Figure 5 (a) and (b). We applied expression balancing to FIR calculation and gain calculation. We unrolled the innermost loop of the FIR initialization and FIR shifting as shown in Figure 5 (c). These two optimizations reduced the number of clock cycles from 41250 to 29730 , and then from 29730 to 20785 . The area before and after restructuring code did not change significantly. The number of BRAMs and DSP48 remained same, and number of FF and LUT decreased slightly. All designs had the same clock period. In general, optimizing the code in software resulted in a 59\%(20215 to 20785) reduction in the number of clock cycles. Due to code restructuring, $\mathrm{C}$ code was reduced to 700 lines to 600 lines.

ChannelFunction: Once again this module did not require much optimization since we coded this module with restructured way. This module uses 7 BRAM, 56 DSP, 4448 FF, and 4968 LUT.

\section{Bit-Width Optimization}

Bit-width optimization impacts both throughput and area. We manually calculated the required bits for all major variables and arrays. We achieved this by calculating values of variables and arrays using floating type. Then we find out needed bit width for each variable by analyzing the max/min range for each variable. Then using the AutoESL's internal arbitrary precision integer and fixed-point data types (ap_int, ap_fixed), we defined new types. This step required more time and effort than any other step.

PathDelays: We applied bit-width optimization on the restructured code. This reduced DSP48E, FF and LUT by $51 \%$ (39 to 19 ), $89 \%$ (4070 to 424 ), $87 \%$ (4631 to 563 ) respectively from the previous stage (Restructured). It also increased the throughput by $90 \%$ due to decreased number of clock cycles from 2000 to 191. The number of BRAMs remained same.

PathGains: We performed bit-width optimization on top of restructured code for the PathGains module. PathGains is largest module, and bit-width optimization took a substantial amount of time. This optimization increased throughput by $56 \%$. Again the throughput increase is resulted from decrease in number of clock cycles from 20785 to 7967 . Bit-width optimization reduced the number of BRAMs, DSP48E, FF and LUT by $50 \%$ (84 to 42 ), $58 \%$ (112 to 47 ), $69 \%$ (67088 to 20399 ), and $65 \%$ (64176 to 22121 ) respectively.

ChannelFunction: Finally, we applied bit-width optimization to ChannelFunction. It decreased number of clock cycles from 902 to 529 which resulted in increased throughput by $40 \%$. This step also reduced the number of DSP48E, FF and 
LUT by $78 \%$ (56 to 12 ), $84 \%$ (4448 to 702 ), and $79 \%$ (4968 to 994$)$ respectively. Number of BRAM remained same.

\section{E. Pipelining/Unrolling/Partitioning (PUP)}

AutoESL provides a pipeline directive to pipeline functions and loops. AutoESL partitions small arrays inside the region and unrolls the loop where the pipeline directive is used. If there are large arrays (not automatically partitionable), then the user can specify that they be divided into separate memories (BRAMs) or as registers. AutoESL's dataflow directive performs coarse grain pipelining at the function and loop level. The dataflow directive increases concurrency between functions and loops by executing the function or loop as soon as the data is available. Thus, it creates a memory between the blocks. Currently, the dataflow directive has limited capabilities. For example, it can be used where data is accessed in a FIFO order. We tried using the dataflow directive in our designs, and it significantly reduced the performance (by a factor of approximately 40X); it did result in a smaller design, but overall this tradeoff was not beneficial. Therefore we did not use the dataflow directive in our final design.

We describe the specific optimizations that we performed on each of the modules in more detail in the following.

PathDelays: We applied pipeline and partiiton on the bitwidth optimized code. We partitioned 5 BRAMs into registers. In AutoESL, complete partitioning a BRAM results discrete registers. We used one pipeline directive on top of function with initiation interval (II) $=1$. The final design is shown in Figure 3. Our design had 4 clock cycles with 5.394 ns of clock period. Since partitioning BRAM and pipelining increase amount of parallelism, it also increased area. The number of DSP48E, FF and LUT increased by 57\% (19 to 30), 85\% (424 to 786 ), and $651 \%$ (563 to 4230 ).

PathGains: Since large BRAMs limit the pipelining, we partitioned the BRAMs that are accessed frequently within a pipelined region. We partitioned 12 of the 42 BRAMs by mapping them to registers. We pipelined the entire function, thus all of the loops were unrolled. This increased the throughput by $93 \%$ due to decreased number of clock cycles from 7967 to 501. The area also increased due to duplicated hardware resources. The BRAMs decreased by 12 since moved this data into registers. DSP48E usage increased by $82 \%$ (47 to 86 ), FF increased by $68 \%$ (20399 to 34421 ), and LUT increased by $75 \%$ (22121 to 38893$)$.

ChannelFunction: We applied pipelining with an $\mathrm{II}=1$ and partitioned seven BRAMs in ChannelFunction. This improved throughput by $98 \%$ and resulted higher area. DSP48E usage increased from 12 to 40, FFs increased from 702 to 2392 and LUTs increased from 994 to 5008.

\section{RESUlts}

The experimental results are obtained using Xilinx AutoESL (Version 2011.1) and ISE (Version 13.1). For each design we report the number of BRAM, DSP, FF, LUT, and the latency. The latency is calculated by multiplying number of clock cycles with clock period. We compare the latency of the software version against the hardware for each design. Then we present the speedup of final hardware version over optimized software version. For each experiment we target an xc6vlx240t FPGA.

\section{A. Verification/Integration}

After applying each optimization, we verify the correctness of each hardware design against the original software using the AutoESL simulator (autosim). After verifying the correctness of each module in each step, we integrate the final optimized version of the three modules from PUP stage to make a complete emulator. This is done to understand how the HLS tools handle large designs. Theoretically, the tools should be able to optimize the complete design in an optimal manner. But this is not always possible due to the complexities of the HLS problems. There are two different ways to integrate three sub modules into one.

Integration using AutoESL: In this case, we create a top level function in $\mathrm{C}$ (which we call emulator) for the three modules. Then we synthesize the emulator with the optimization pragmas from the PUP stage. This may create a slower and larger design if AutoESL is unable to correctly optimize the resulting large data flow graph in an optimal manner.

Manual Integration: Here, we create a top module in Verilog and manually integrate the optimized designs for PathDelays, PathGains and ChannelFunction. Then we synthesize the top module using ISE. It is a divide and conquer method using a hand partitioned method for synthesizing the sub modules.

The block diagram of the emulator is shown in Figure 11. In the integrated design, we use two different clocks. One clock for PathGains which is $100 \mathrm{Mhz}$, and other clock for PathDelays and ChannelFunction which is $151 \mathrm{Mhz}$. We use two different frequencies as described in Table II. The modules PathGains and PathDelays run in parallel and their outputs (gain, weight, indexstart) are consumed by ChannelFunction function to calculate SampleOut complex signal.

The PathDelays module calculates indexstart and weight. In each case four and six clock cycles, respectively, are required to calculate the SampleOut signal. This requires a total 62 ns is needed to calculate SampleOut with 10 clock cycles at $151 \mathrm{Mhz}(151 \mathrm{Mhz}=6.62 \mathrm{~ns}$ period, $10 \times 6.62=62 \mathrm{~ns})$. The PathGains runs slowest and it outputs results every $4994 \mathrm{~ns}$ (0.2 Mhz). In our statistical channel model, we use the same result of the PathGains (same gains) for every 80 SampleOut calculation. (PathGains runs in 0.2 Mhz (4994 ns and 4994/62 ns $=80$ ). The PathGains module writes output to registers. We represented it with FIFO as shown in the Figure 11.

We verified the correctness of emulator of both designs. The input to the emulator are the sample input signal, carrier frequency, receiver speed, LOS gain, and path velocities. The output of the emulator is the sampled output signal in a complex form.

\section{B. Experimental Results}

We present area results first. We present this in two figures. The first shows the number of BRAM with DSP (Figure 6). 
TABLE I

DeVice Utilization CHARACTERISTICS FOR PATHDElays, PATHGains AND CHANNELFUNCTION FOR FIVE PATHS.

\begin{tabular}{|l|l|l|l|l|l|}
\hline & Slices & LUT & FF & DSP48E & BRAM \\
\hline PathDelays & 584 & 1843 & 411 & 30 & 0 \\
\hline PathGains & 2783 & 8759 & 7044 & 53 & 30 \\
\hline ChannelFunction & 1131 & 3469 & 1798 & 40 & 0 \\
\hline
\end{tabular}

TABLE II

CLOCK CYCLES, CLOCK PERIOD AND LATENCY OF EACH SUB MODULE.

\begin{tabular}{|l|l|l|l|}
\hline & $\begin{array}{l}\text { Clock } \\
\text { cycles }\end{array}$ & $\begin{array}{l}\text { Clock period (ns)/ } \\
\text { Frequency }\end{array}$ & $\begin{array}{l}\text { Latency(ns)/ } \\
\text { Throughput }\end{array}$ \\
\hline PathDelays & 4 & $5.394 / 185 \mathrm{Mhz}$ & $21 / 47 \mathrm{Mhz}$ \\
\hline PathGains & 501 & $9.97 / 100 \mathrm{Mhz}$ & $4994 / 0.2 \mathrm{Mhz}$ \\
\hline ChannelFunction & 6 & $6.62 / 151 \mathrm{Mhz}$ & $37 / 26 \mathrm{Mhz}$ \\
\hline
\end{tabular}

TABLE III

DEVICE UTILIZATION CHARACTERISTICS OF COMPLETE EMULATOR FOR EMULATORS INTEGRATED BY AUTOESL AND MANUALLY.

\begin{tabular}{|l|l|l|l|l|l|}
\hline & Slices & LUT & FF & DSP & BRAM \\
\hline Emulator (Manual) & 5020 & 14221 & 9564 & 123 & 30 \\
\hline Emulator (AutoESL) & 14283 & 47190 & 30691 & 70 & 60 \\
\hline
\end{tabular}

The second displays FF with LUT (Figure 7). This is essentially a graphical recap of the optimizations described in the previous sections. ChannelFunction has same code for baseline and restructured designs.

One of the biggest application tradeoffs that we explored is the number of paths per channel. This highly depends on the application scenario. Some situations require fewer paths than others. For example, communication in a city, or mountainous area typically require more path to accurately model the channel due to reflections of the signal. The same is true for indoor communications. Our model uses five paths as a default parameter; other common models such as Stanford model and ITU model use three paths and six paths [15], [16].

A big advantage of HLS is design space exploration. In this case it allows us set parameters such as number of paths using minimal modifications to the $\mathrm{C}$ code, and synthesizing the architecture to determine its design metrics. We studied how different paths change the hardware implementation. Figure 8 shows how the area (in number of slices) changes as the number of paths increase. In general it is linear relationship, e.g., an implementation with five paths is $5 \times$ larger than the design with one path. In Figure 8, PathGains does not have $5 \times$ relationship due to constant area of the CORDIC core. The throughput does not change since the computation required by adding the different paths is for the most part independent, and thus can be parallelized. All of the other reported results use five paths. Table I shows more detailed area results of PathDelays, PathGains and ChannelFunction for five path implementation. The slice results are equivalent to the rightmost three bars in Figure 8.

Figure 9 compares the latency of the software implementation with the latency of four designs (Baseline, restructured,

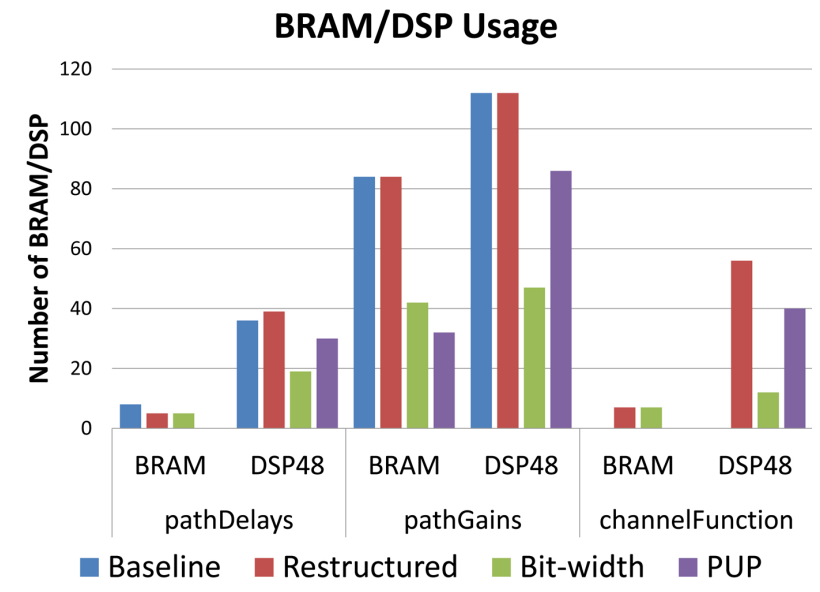

Fig. 6. Number of BRAM/DSP for Baseline, Restructured, Bit-width and PUP optimizations for PathDelays, PathGains and ChannelFunction.

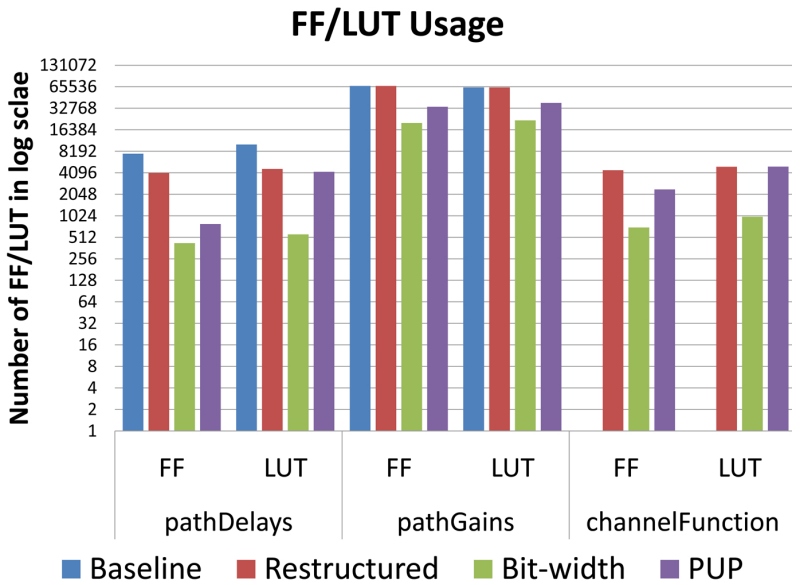

Fig. 7. Number of FF/LUT for Baseline, Restructured, Bit-width and PUP optimizations of PathDelays, PathGains and ChannelFunction.

bit-width and PUP). The latency of the software is measured on a Intel i7 $3.4 \mathrm{GHz}$ multi-core machine. We applied OpenMP on top of restructuring code to get optimized software implementation. We compared the hardware performance against the best optimized software performance. The optimized software version of PathDelays and PathGains has 29X and 18X times better performance than initial software. This is shown in Figure 10. The module ChannelFunction has little parallelism and the computation in module ChannelFunction is not so complex. Thus, there was little room for the optimization of ChannelFunction. We used the same ChannelFunction implementation for all of our comparisons.

In general, as shown in the Figure 9, hardware implementations corresponding to baseline and restructured versions are slower than the optimized software version. This is due to the clock cycle difference between CPUs and FPGAs. For example, the baseline implementations are slower than the software equivalents by a factor of 106X, 17X and $4.8 \mathrm{X}$ of PathDelays, PathGains, and ChannelFunction.

In Figure 9, the final hardware performance results ob- 


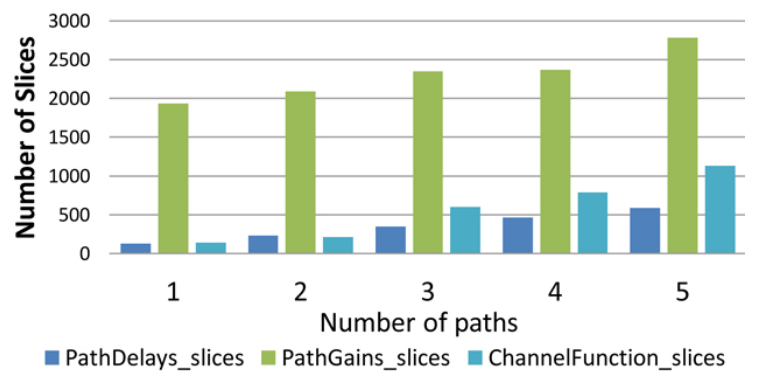

Fig. 8. Slice numbers for scaling number of paths

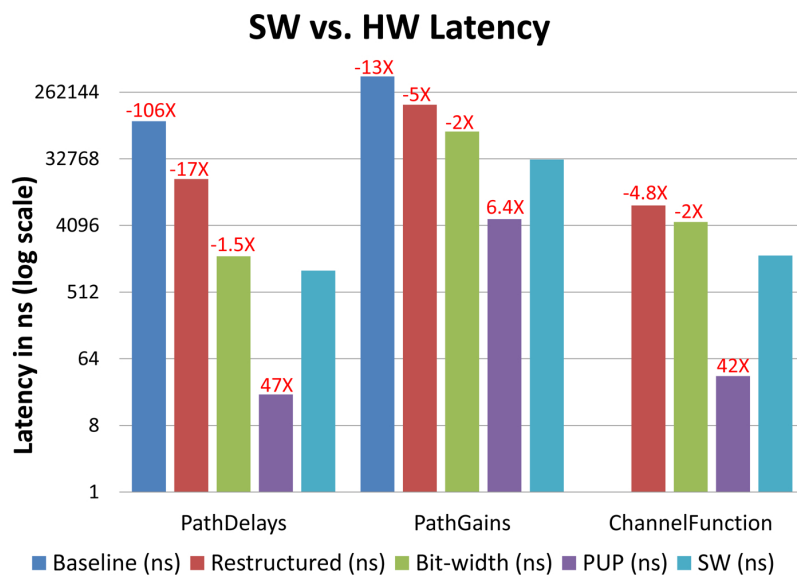

Fig. 9. Software versus hardware latency for Baseline, Restructured, Bit width and PUP optimizations of PathDelays, PathGains and ChannelFunction. Negative $(-X)$ means slower by $X$ than software(SW). Positive $X$ means faster by $\mathrm{X}$ than software.

tained by PUP optimization are compared to the software performance. The hardware results are better by $46 \mathrm{X}, 6 \mathrm{X}$ and $42 \mathrm{X}$ over the equivalent software version. PathGains gave the worst speedup. This is largely due to the fact that it uses CORDIC which requires 79 cycles thus limiting the initiation interval. The comparison of the baseline software code with our final hardware implementation has significantly better results; the hardware implementation of PathDelays and PathGains is 200,000X and 2000X faster respectively. In other words, restructuring the code gives significant software performance benefits.

Table II presents the number of clock cycles, clock period (frequency) and latency of each sub module. The integrated emulator runs in $62 \mathrm{~ns}(16 \mathrm{Mhz})$. This is $41 \mathrm{X}$ times faster than software emulator. The software emulator uses the same optimized sub modules.

In the first row of Table III, we present area results for manually integrated version of emulator. In the second row of Table III, we present the area results for AutoESL based integrated version of the emulator. The AutoESL based integration has $5 \mathrm{X}$ larger area than manual integrated one while being $2 \mathrm{X}$ times slower than manually integrated emulator.

We spent a total of five weeks to design the emulator. Of that, three weeks went towards understanding the application and writing the restructured code. Two weeks was spent

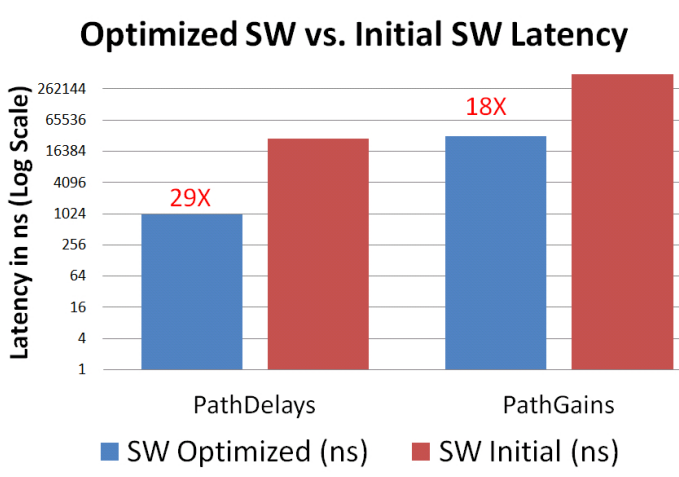

Fig. 10. Performance of initial and optimized versions of software for PathDelays and PathGains.

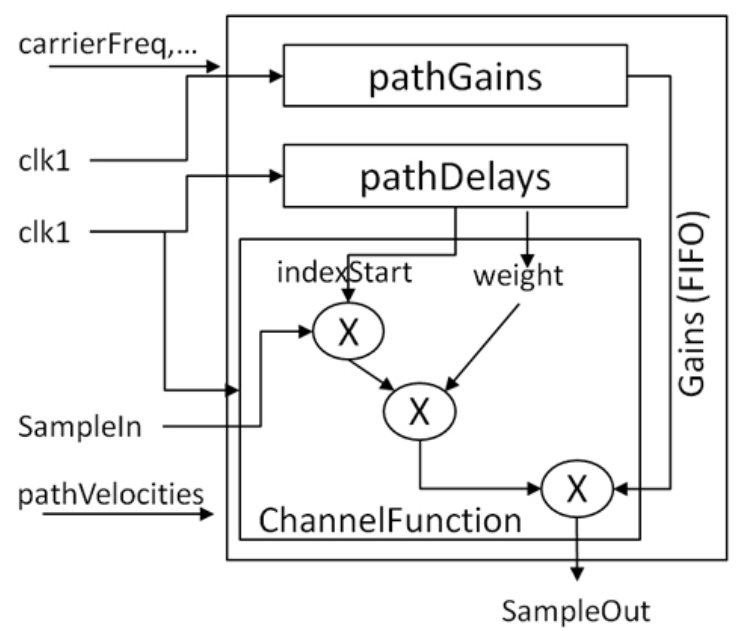

Fig. 11. Block diagram of the emulator.

performing bit-width optimization and PUP with the majority of this time spent on bit-width optimization.

\section{Discussion}

Quality of Result: The ultimate way to compare the results from AutoESL is to compare the final optimized design with hand coded version. This is a difficult endeavor and one that we would argue is not totally necessary. HLS tools are reaching the point where if you code the input design appropriately, then you will get a design close to if you designed it from RTL. And the HLS tools allows you to quickly to change the architecture to see its effects. This is not to say that HLS tools are a panacea. In fact, we hope this article relays the fact that HLS tools require a good understanding of how the hardware and the synthesis process works. At this point these tools are still far from giving great results on code that was designed by a software programmer. The user of these tools needs to understand how the tool will synthesize the final architecture in order to get the best results.

Previous research [1], [2], [3] designed different models and use different fading and use different parameters. To our best knowledge, this is the first work that implements statistical 
model. The emulator by Iskander et al. [4] only gives a software version in Matlab. Our performance results are thousands of times better than this Matlab version. Another way to justify the result is we estimate the optimal performance and area for each module. However, the estimation may not be accurate for larger modules such as PathGains. Thus it may not be fair comparison. Therefore, it is not possible to do fair comparison with other hand coded designs at this point.

We studied other works that used AutoESL. In order to get reasonable performance/area results with HLS tools, designers write manual $C$. AutoESL does not generate an optimal HDL from sequential software $\mathrm{C}$ magically. The flow of design is same as traditional RTL design flow such as they create architecture, writes a $\mathrm{C}$ code targeting the architecture. That means designers write $\mathrm{C}$ code instead of Verilog/VHDL and follow same steps normal RTL designer follows. For example, recently several research works used AutoESL and presented competitive result with hand coded RTL [7], [6].

Lessons learned: Our experience shows that achieving the target performance and area depends a lot on the code size, the quality of the restructured code and the application. We spent a relatively small amount time achieving the target goal for the smaller modules, i.e., those with fewer lines of code. We spent a disproportionate amount of time optimizing PathGains as it contained significantly more lines of code. Initially, we did not restructure the PathGains code well (we achieved $25 \mathrm{kHz}$ with poor restructuring). Then we spent time deeply analyzing the code, e.g., drawing sample hardware architectures, manually performing some design space exploration to derive different micro architectures. This gave us insight into what to optimize and how. An important lesson is HLS tools require that the user understands what architectures are being generated. And while they allow you to more quickly get to a specific RTL implementation, a good design requires someone who deeply understands what the optimizations are doing. This is especially true for larger applications with tight constraints (i.e., PathGains).

Another major issue that we faced was the synthesis times for the larger applications. In our initial trials at restructuring the PathGains module, AutoESL would run for hours, run out of memory and then crash. Better optimized designs solved this problem; therefore the synthesis time is not necessarily a function of the number of lines of code rather a function of how many lines of RTL code the HLS tool creates.

Final lesson is HLS may give very poor design in initial stage. The initial results are somewhat very conservative and getting better design highly depends on designer's ability to understand the application.

\section{CONCLUSION}

In this paper, we presented the design and implementation of wireless channel emulator using high-level synthesis tool (AutoESL). We described the underlying model of our wireless channel emulator. We developed an FPGA implementation of the emulator that met the design constraints for a single channel. We studied how the various high-level synthesis tool optimizations affect the area and performance results. As a future work, we plan to extend current single channel wireless emulator to multi channel wireless emulator.

\section{REFERENCES}

[1] K. Borries, G. Judd, D. Stancil, and P. Steenkiste, "Fpga-based channel simulator for a wireless network emulator," in Vehicular Technology Conference, 2009. VTC Spring 2009. IEEE 69th. IEEE, 2009, pp. 1-5.

[2] P. Murphy, F. Lou, A. Sabharwal, and J. Frantz, "An fpga based rapid prototyping platform for mimo systems," in Signals, Systems and Computers, 2003. Conference Record of the Thirty-Seventh Asilomar Conference on, vol. 1. IEEE, 2003, pp. 900-904.

[3] S. Buscemi and R. Sass, "Design of a scalable digital wireless channel emulator for networking radios," in MILITARY COMMUNICATIONS CONFERENCE, 2011-MILCOM 2011. IEEE, 2011, pp. 1858-1863.

[4] C. Iskander and H. Multisystems, "A matlab-based object-oriented approach to multipath fading channel simulation," MATLAB White Paper, 2008.

[5] J. Cong, B. Liu, S. Neuendorffer, J. Noguera, K. Vissers, and Z. Zhang, "High-level synthesis for fpgas: From prototyping to deployment," Computer-Aided Design of Integrated Circuits and Systems, IEEE Transactions on, vol. 30, no. 4, pp. 473-491, 2011.

[6] J. Chen, J. Cong, M. Yan, and Y. Zou, "Fpga-accelerated 3d reconstruction using compressive sensing," in Proceedings of the ACM/SIGDA international symposium on Field Programmable Gate Arrays. ACM, 2012, pp. 163-166.

[7] K. Rupnow, Y. Liang, Y. Li, D. Min, M. Do, and D. Chen, "High level synthesis of stereo matching: Productivity, performance, and software constraints," in Field-Programmable Technology (FPT), 2011 International Conference on. IEEE, 2011, pp. 1-8.

[8] J. Noguera, S. Neuendorffer, S. Van Haastregt, J. Barba, K. Vissers, and C. Dick, "Implementation of sphere decoder for mimo-ofdm on fpgas using high-level synthesis tools," Analog Integrated Circuits and Signal Processing, pp. 1-11, 2011.

[9] K. Vissers, S. Neuendorffer, and J. Noguera, "Building real-time hdtv applications in fpgas using processors, axi interfaces and high level synthesis tools," in Design, Automation \& Test in Europe Conference \& Exhibition (DATE), 2011. IEEE, 2011, pp. 1-3.

[10] A. Takach, B. Bowyer, and T. Bollaert, "C based hardware design for wireless applications," in Proceedings of the conference on Design, Automation and Test in Europe-Volume 3. IEEE Computer Society, 2005, pp. 124-129.

[11] M. Myllyla, M. Juntti, and J. Cavallaro, "Architecture design and implementation of the increasing radius-list sphere detector algorithm," in Acoustics, Speech and Signal Processing, 2009. ICASSP 2009. IEEE International Conference on. IEEE, 2009, pp. 553-556.

[12] B. Buyukkurt and W. Najj, "Compiler generated systolic arrays for wavefront algorithm acceleration on fpgas," in Field Programmable Logic and Applications, 2008. FPL 2008. International Conference on. IEEE, 2008, pp. 655-658.

[13] J. Villarreal and W. Najjar, "Compiled hardware acceleration of molecular dynamics code," in Field Programmable Logic and Applications, 2008. FPL 2008. International Conference on. IEEE, 2008, pp. 667670.

[14] J. Xu, N. Subramanian, A. Alessio, and S. Hauck, "Impulse c vs. vhdl for accelerating tomographic reconstruction," in Field-Programmable Custom Computing Machines (FCCM), 2010 18th IEEE Annual International Symposium on. IEEE, 2010, pp. 171-174.

[15] V. Erceg, K. Hari, M. Smith, D. Baum, K. Sheikh, C. Tappenden, J. Costa, C. Bushue, A. Sarajedini, R. Schwartz et al., "Channel models for fixed wireless applications," 2001.

[16] I.-R. R. M.1225, "Guidelines for evaluation of radio transmission technologies for imt-2000," 1997. 